\title{
Organic electronics for precise delivery of neurotransmitters to modulate mammalian sensory function.
}

\author{
Daniel T Simon, Sindhulakshmi Kurup, Karin C Larsson, Ryusuke Hori, \\ Klas Tybrandt, Michel Goiny, Edwin W H Jager, Magnus Berggren, \\ Barbara Canlon and Agneta Richter-Dahlfors
}

\section{Linköping University Post Print}

N.B.: When citing this work, cite the original article.

Original Publication:

Daniel T Simon, Sindhulakshmi Kurup, Karin C Larsson, Ryusuke Hori, Klas Tybrandt, Michel Goiny, Edwin W H Jager, Magnus Berggren, Barbara Canlon and Agneta RichterDahlfors, Organic electronics for precise delivery of neurotransmitters to modulate mammalian sensory function., 2009, Nature Materials, (8), 9, 742-746.

http://dx.doi.org/10.1038/nmat2494

Copyright: Nature Publishing Group http://npg.nature.com/

Postprint available at: Linköping University Electronic Press http://urn.kb.se/resolve?urn=urn:nbn:se:liu:diva-20279 


\section{Organic electronics for precise delivery of neurotransmitters to modulate mammalian sensory function}

Daniel T. Simon ${ }^{1,4}$, Sindhulakshmi Kurup ${ }^{2,4}$, Karin C. Larsson ${ }^{2,4}$, Ryusuke Hori, ${ }^{3, \text {, }}$, Klas Tybrandt $^{1,4}$, Michel Goiny ${ }^{3}$, Edwin W. H. Jager ${ }^{1,4}$, Magnus Berggren ${ }^{1,4}$, Barbara Canlon ${ }^{3}$, Agneta Richter-Dahlfors ${ }^{2,4, *}$

${ }^{1}$ Department of Science and Technology (ITN), Linköping University, Norrköping, Sweden.

${ }^{2}$ Department of Neuroscience and ${ }^{3}$ Department of Physiology and Pharmacology, Karolinska Institutet, Stockholm, Sweden.

${ }^{4}$ Strategic Research Center for Organic Bioelectronics (OBOE), Linköping and Stockholm, Sweden.

Significant advances have been made in the understanding of the pathophysiology, molecular targets, and therapies for the treatment of a variety of nervous system disorders. Particular therapies involve electrical sensing and stimulation of neural activity ${ }^{1-4}$, and significant effort has therefore been devoted to the refinement of neural electrodes ${ }^{5-8}$. However, direct electrical interfacing suffers from some inherent problems, e.g., the inability to discriminate amongst cell types. Thus, there is a need for novel devices to specifically interface nerve cells. Here we demonstrate an organic electronic device capable

\footnotetext{
${ }^{\mathbb{I}}$ Present address: Department of Otolaryngology, Head \& Neck Surgery, Graduate School of Medicine, Kyoto University, Japan.

* Correspondance should be addressed to A.R-D. (agneta.richter.dahlfors@ki.se).
} 
of precisely delivering neurotransmitters in vitro and in vivo. In converting electronic addressing into delivery of neurotransmitters, the device mimics the nerve synapse. Using the peripheral auditory system, we show that out of a diverse population of cells, the device can selectively stimulate nerve cells responding to a specific neurotransmitter. This is achieved via precise electronic control of electrophoretic migration through a polymer film. This mechanism provides several sought-after features for regulation of cell signalling: $i$ ) exact dosage determination via electrochemical relationships; $i i)$ minimally disruptive delivery due to lack of fluid flow; and, iii) on-off switching. This technology has great potential as a therapeutic platform and could help accelerate the development of therapeutic strategies for nervous system disorders.

In order to develop a programmable "machine-to-brain" communication platform, numerous criteria must be met, e.g., dosage precision, and dynamic spatial and temporal control. Furthermore, delivery should preferably be achieved with minimal fluid flow, as liquid flow can lead to undesirable effects, e.g., chemical gradient disruption ${ }^{9}$, or increased pressure in compartments with limited volume. Non-convective delivery would enable introduction of biosubstances per se into small compartments in organs, e.g., the cochlea. Organic conjugated polymers have been investigated in recent years as delivery platforms that fulfil many of these requirements, in part due to their chemical and material properties which facilitate their incorporation into bio-medical devices ${ }^{10,11}$. As volume-change actuators, they have been explored as reversible valves exposing drug-loaded microreservoirs ${ }^{12}$. This electrochemical actuation has also been investigated for the release of drugs from the polymer itself ${ }^{13,14}$. Abidian et al. have even demonstrated controlled release from conducting polymer-coated drug-loaded nanofibers fabricated directly on a neural implant electrode ${ }^{15}$.

Each of these technologies takes advantage of the role of ions in polymer conduction. Electronic and ionic conduction coexist - and are often coupled - in conducting polymer systems ${ }^{16,17}$. Indeed, the fact that electronic conduction can be ion mediated leads to the 
conclusion that these materials could provide an ideal interface for signal transduction between electron- and ion-mediated systems. This concept was recently demonstrated in an organic electronic "ion pump"18. When addressing the device with a driving voltage, $\mathrm{K}^{+}$was electrophoretically transported from a source electrolyte, through a film of the conducting polymer ${ }^{19}$ poly(3,4-ethylenedioxythiophene) doped with poly(styrenesulfonate) (PEDOT:PSS) ${ }^{20-}$ ${ }^{22}$, into a target reservoir containing neuronal cells. Local increase of extracellular $\left[\mathrm{K}^{+}\right]$ depolarized the cell membrane, thereby activating membrane-bound $\mathrm{Ca}^{2+}$ pumps, i.e., the electronic input signals were translated into ion fluxes and, in turn, activation of intracellular signal transduction pathways.

This technology, based on simple monolithic design, realizes the criteria stated above. Delivery is achieved with minimal physiological disturbances as electronic signals are translated into ion transport in the absence of fluid flow. Spatial control is defined by photolithographic patterning $^{18}$ (Supplementary Information), whereas temporal control is achieved by modulation of the electrophoretic driving voltage. The latter provides the possibility of programming the ion transport to mimic dynamic temporal patterns, e.g., the oscillations characteristic of $\mathrm{Ca}^{2+}$ signaling ${ }^{23}$.

To analyze whether we could expand the repertoire of signal substances transported through the electronic matrix, we tested the neurotransmitters glutamate (Glu), aspartate (Asp), and $\gamma$-amino butyric acid (GABA). These small molecules exist as positively charged ions depending on the $\mathrm{pH}$, which is required for the electrophoretic action of the ion pump. Glu is the major excitatory neurotransmitter in the central nervous system (CNS) where it mediates fast synaptic transmission. Together with its receptors, Glu plays critical roles in synaptic plasticity, basic processes of memory and learning, brain development, as well as neurological and psychiatric disorders ${ }^{24}$. Asp is also an excitatory neurotransmitter, whereas GABA is the major inhibitory neurotransmitter in the mammalian CNS. The pathophysiology of numerous 
neuropsychiatric disorders, including anxiety and depression, are suggested to be due to disturbances in the GABA system ${ }^{25}$.

We prepared planar ion pump devices, comprising a single, biocompatible PEDOT:PSS layer, as previously reported $^{18}$. The single film is divided by an electronically insulating - but still ionically conducting - region (Figure 1 and Supplementary Information). When voltage is applied, an electrochemical circuit is established, leading to oxidation of the source electrode (anode, Eqn. 1), and reduction of the target electrode (cathode, Eqn. 2), where $\mathrm{M}^{+}$is the cation present in the source electrolyte.

$$
\begin{aligned}
& \text { PEDOT }^{0}+\mathrm{M}^{+}: \mathrm{PSS}^{-} \rightarrow \text { PEDOT }^{+}: \mathrm{PSS}^{-}+\mathrm{M}^{+}+\mathrm{e}^{-} \\
& \text {PEDOT }^{+}: \mathrm{PSS}^{-}+\mathrm{M}^{+}+\mathrm{e}^{-} \rightarrow \text { PEDOT }^{0}+\mathrm{M}^{+}: \mathrm{PSS}^{-}
\end{aligned}
$$

The cations are electrophoretically transported through the region of film joining the two electrodes, then enter the cathodic side of the film, where they are delivered into the electrolyte. This structure of electronically conducting electrodes separated by an electronically insulating channel allows for application of a wide range of voltages - in excess of $30 \mathrm{~V}$ - without excessive electric fields in the target system. Furthermore, the use of PEDOT:PSS alleviates problems associated with secondary electrochemical reactions at such elevated voltages (Supplementary Information). 

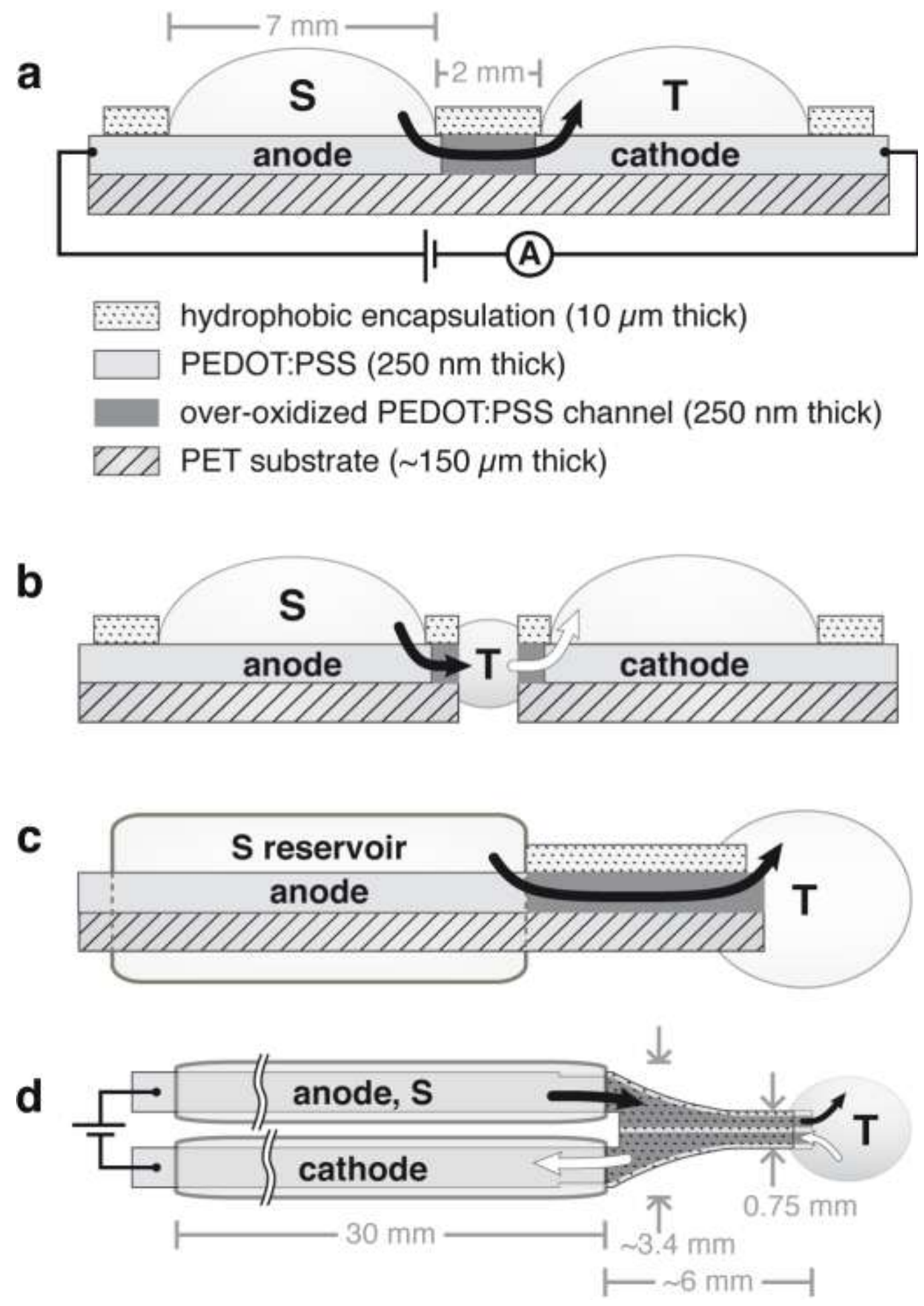

Figure 1 Planar and encapsulated geometries of the delivery device. a, Side view of the planar device used in initial Glu, Asp, and GABA transport studies. The black arrow indicates the flow of charged neurotransmitters from the source electrolyte, S, through the anode, then through the over-oxidized channel, and finally out into the target electrolyte, $\mathrm{T}$, via the cathode. Layer thicknesses are indicated in the material legend. b, Side view showing the developmental progression from the planar device (a), to the planar device with intermediate electrolyte (salt bridge) - i.e., the effective target system T. Here, the white arrow indicates the flow of arbitrary positively charged species from $\mathrm{T}$ into the cathodic electrolyte. c, Side view scheme of the encapsulated device. Here, only the source/anode system is depicted with cylindrical encapsulation. The arrow again indicates ion flow. d, Top view of the encapsulated device, showing both electrolyte chambers and the requisite target system $\mathrm{T}$. The arrows are analogous to those in (b). The electrolyte reservoir tubes are $2 \mathrm{~mm}$ in outer diameter. 
Using Glu, Asp, and GABA as source electrolytes, the delivery capabilities of the device were demonstrated at multiple driving voltages for a variety of times, with individual devices used for each parameter tested (Figures 2a-c). By comparing the integrated electronic current to the quantity of neurotransmitters delivered into the target electrolyte (Supplementary Information), a transport efficiency can be defined by the electron:molecule ratio. This ratio is precisely $2.7 \pm 0.2$ for Glu $(n=16), 6.3 \pm 0.5$ for Asp $(n=10)$, and $1.3 \pm 0.1$ for GABA $(n=10)$ (value \pm s.d.). For materials with low $\mathrm{pK}_{\mathrm{a}}$, i.e., Glu and Asp, the excess protons present in the source solution will also be pumped. Owing to the smaller size of protons compared to Glu or Asp, their mobility through the channel can be significantly higher, explaining the larger electron:molecule ratios for Glu and Asp. The transport rate can be tuned by the operating voltage, providing a full range of transport up to quantities on the order of $100 \mu M$ in the total liquid volume. Locally however, the concentration can be significantly higher before the molecules diffuse away ${ }^{23}$. The approximate concentration of Glu in the synaptic cleft has been reported to be $2-1000 \mu M^{26}$, thus the device operates in a relevant physiological range.

The lifetime of the device is limited by the amount of oxidizable PEDOT (cf. Eqn. 1) and can thus be tailored by the electrode dimensions (Supplementary Information). The present electrode geometry allows for device operation on the order of $1 \mathrm{~h}$ in constant pumping mode, i.e., constant current. The PEDOT could also be returned to a more reduced state, e.g., by reversing the voltage. Utilising pulsed delivery ${ }^{23}$, the lifetime should be bounded only by the concentration of molecules in the source electrolyte and could therefore be dramatically extended. Figure $2 \mathrm{~d}$ illustrates that the delivery rate is stable after an initial equilibration period corresponding to the time required to fill the channel with the intended ions on first usage. 

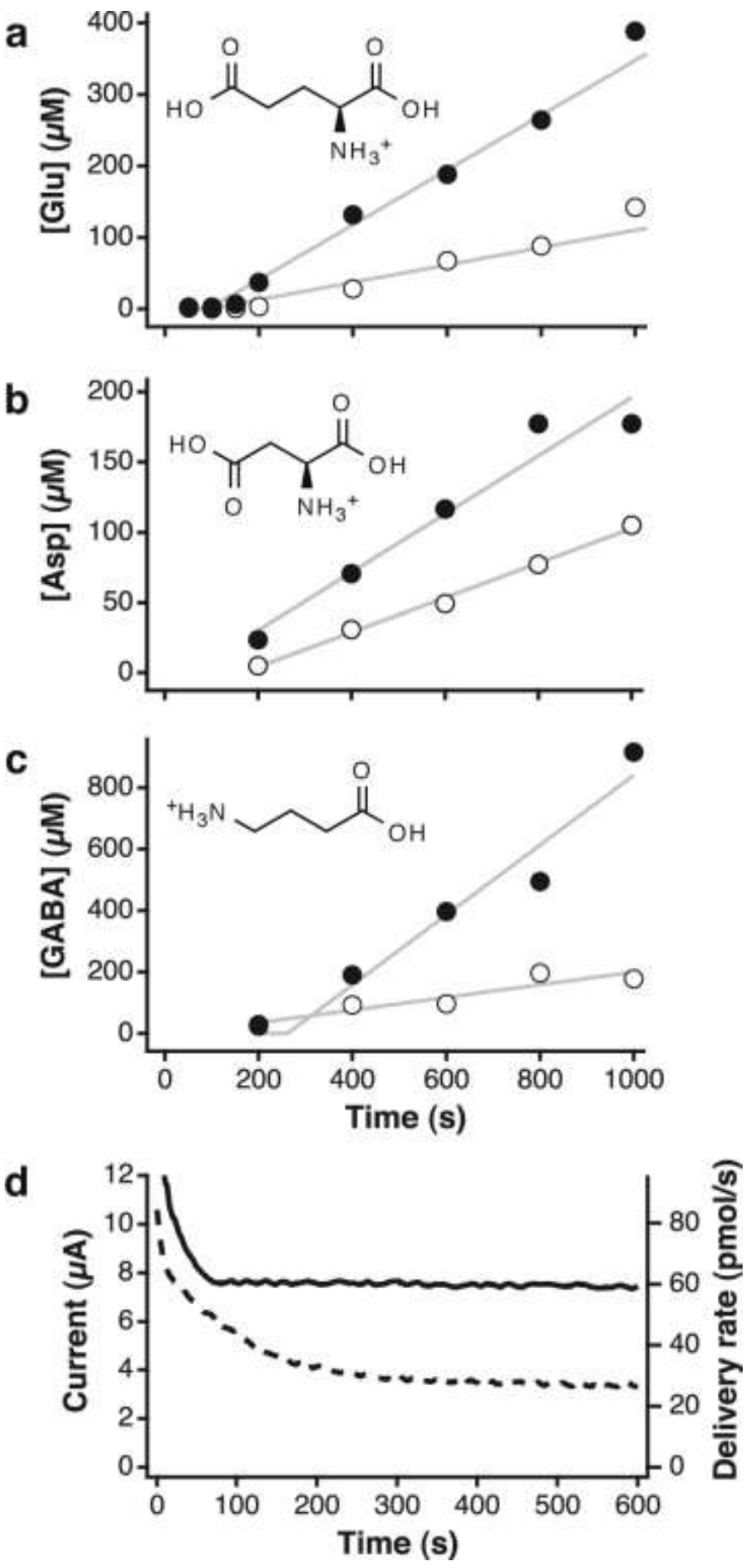

Figure 2 Transport of neurotransmitters in the planar delivery device. Concentration of $\mathbf{a}, \mathrm{Glu}$ (148 g/mol), b, Asp (134 g/mol), and c, GABA $(104 \mathrm{~g} / \mathrm{mol})$, respectively, in the target electrolyte $(150 \mu \mathrm{l})$ as a function of time, where the device was operated at $4 \mathrm{~V}$ (open symbols) and $8 \mathrm{~V}$ (filled symbols). The gray lines are linear fits with non-zero time offset (Supplementary Information). The insets show the chemical structures of the materials in their positively charged form. d, Representative current vs. time data at $4 \mathrm{~V}$ (dashed line) and 8 $\mathrm{V}$ (solid line) with equivalent delivery rate on the right axis. The data shown is for GABA delivery. 
The successful transport of neurotransmitters encouraged us to redesign the device into an encapsulated, syringe-like form to enable its use in vivo. The first step in this development was the realization that the channel region could comprise an additional electrolyte (Figure 1b). This geometry could enable the central electrolyte to become the effective target system, with the original target acting simply as the electrochemical cathode. By "folding" this system, and providing encapsulation around the individual electrolytes, a syringe-like device is achieved (Figures 1c,d). The operating principle is similar to the planar device, except that cations from the source are delivered to an external target electrolyte, and cations are extracted from this target region and drawn in toward the cathode system, completing the electrochemical circuit.

To ascertain whether the encapsulated device can be used for cell stimulation similarly to the planar device ${ }^{18}$, in vitro experiments were performed. Astrocytes, a sub-type of glial cells present in the CNS, express the receptor for Glu. Upon binding of Glu, membrane-bound ion channels open immediately, promoting $\mathrm{Ca}^{2+}$ influx ${ }^{27-29}$. Therefore, astrocytes represent an ideal system to monitor Glu activation of cells using real-time single-cell ratiometric $\mathrm{Ca}^{2+}$ imaging. The device was loaded with Glu (source) and $\mathrm{NaCl}$ (cathodic) electrolytes and mounted with the tip in contact with the bottom of a dish, adjacent to primary murine astrocytes. After initial baseline recordings of intracellular $\mathrm{Ca}^{2+}$ with the delivery device in the off-state, Glu transport was activated. A significant increase in intracellular $\mathrm{Ca}^{2+}$ revealed successful delivery of Glu (Figure 3) after an equilibration delay similar to that observed in Figure $2 \mathrm{~d}$. The $\mathrm{Ca}^{2+}$ response declined when the voltage was turned off and cells re-established their basal intracellular $\mathrm{Ca}^{2+}$ level. As a control, Glu was next applied manually using a pipette, resulting in a $\mathrm{Ca}^{2+}$ response of similar magnitude to that induced by Glu delivered from the encapsulated device. Collectively, this substantiates the usability of the device for cell activation, and that Glu retains its potent biological form after transport through the polymer. 


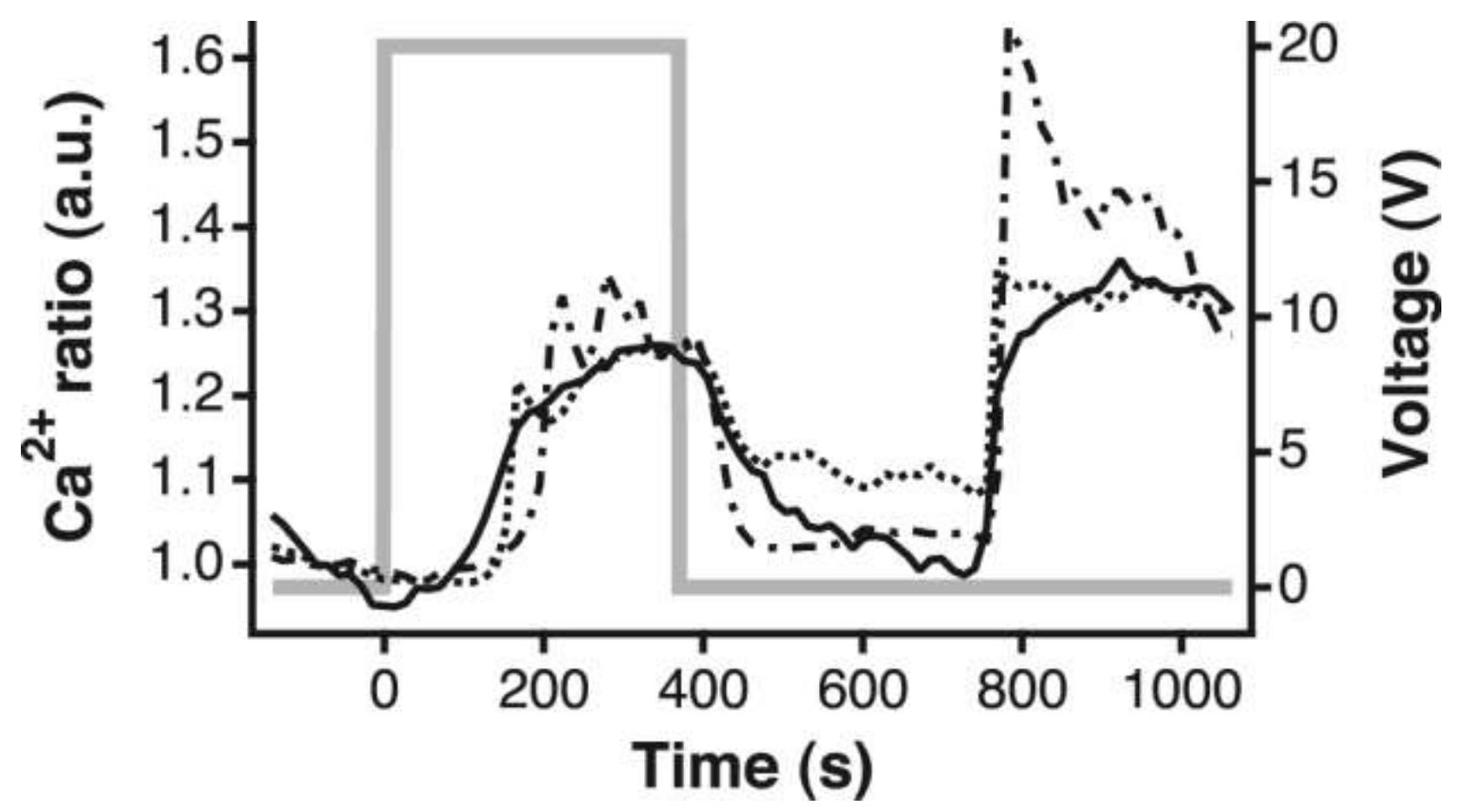

Figure 3 Glu-induced $\mathrm{Ca}^{2+}$ responses in astrocytes. Time-lapse fluorescence microscopy of intracellular $\mathrm{Ca}^{2+}$ recordings in astrocytes induced by Glu delivered via the encapsulated device. Representative tracings from three cells (solid, dotted, and dot-dash lines) in one experiment are shown. The device was switched on by application of voltage for $370 \mathrm{~s}$ (solid gray line), and then switched off. Cells were then allowed to re-establish their basal $\mathrm{Ca}^{2+}$ level. At $730 \mathrm{~s}$, Glu was applied manually using a pipette $\left([\mathrm{Glu}]_{\text {final }}=1 \mathrm{mM}\right)$, eliciting responses of similar magnitude to those from the device-delivered Glu. Experiment was repeated three times.

Having demonstrated how this device converts electronic addressing signals into precise non-convective delivery of Glu, we next investigated its potential use as a novel communication interface between human-made electronics, selective neurotransmission, and brain function. To test the feasibility of this concept, we used the auditory system of the guinea pig as an in vivo experimental platform. Within the cochlea, sound waves of various frequencies are transduced primarily by the inner hair cell system, as opposed to the outer hair cell system ${ }^{30}$. As Glu is the primary neurotransmitter for the inner hair cells, the auditory system can be used to evaluate the ability of the device to target specific cells, i.e., whether the encapsulated ion pump can be used to selectively affect specific cell types in vivo. Excessive Glu is known to exert an excitotoxic effect on the inner hair cells as opposed to the outer hair cells ${ }^{31,32}$, and this pathophysiological effect can be monitored by histological analysis. In addition, excitotoxicity can be analysed in real-time, by monitoring the auditory brainstem response (ABR). Excessive Glu will damage the 
inner hair cells, leading to hearing loss and an attenuated ABR. In the cochlea, high frequency sound waves are transduced at the base, near the round window membrane (RWM), while lower frequencies are transduced towards the apex. Therefore, shifts in ABR threshold (re:pretreatment threshold) at different frequencies indicate, in real-time, the effect of Glu at different distances up the cochlea. Currently, osmotic pumps are utilized to modulate such effects by direct fluid injection ${ }^{33}$. However, the inner hair cells, positioned on a delicate membrane that vibrates with sound stimulation, are highly mechanosensitive, e.g., to disturbances in fluid flow and pressure ${ }^{34}$. To bypass this problem, the ion pump can be used to deliver substances through the RWM, which is an established port of diffusive entry into the cochlea.

The tip of the delivery device was introduced in close proximity to the RWM of anesthetized guinea pigs using conventional ontological surgery (Figure 4a). The procedure is non-invasive to the cochlea, since the device delivers Glu to the outside of the RWM through which Glu enters via diffusion (Figure 4b). Once the device was in place, Glu was delivered continuously for $60 \min (n=5)$ and during this time, ABR shifts were assessed at 0, 15, 30 and $60 \mathrm{~min}$. Within this timeframe, stimulation in the lower basal region (corresponding to $20 \mathrm{kHz}$ transduction), upper basal region $(16 \mathrm{kHz})$, and lower apical region $(8 \mathrm{kHz})$ could be achieved without risking saturation of the entire cochlea with Glu. As the Glu solution was at low $\mathrm{pH}$, control experiments $(n=3)$ delivering only $\mathrm{H}^{+}$were also performed. At 15 min, an ABR shift of only $7.5 \mathrm{~dB}$ was observed for both Glu and $\mathrm{H}^{+}$for all frequencies, indicating the $\mathrm{Glu}$ concentration had yet to reach excitotoxic levels (Figure 4c). At the $30 \mathrm{~min}$ time point, increased ABR shifts ( $10 \mathrm{~dB}$, data not shown) were observed in some cases for Glu while the $\mathrm{H}^{+}$response remained consistently low. After 60 min of Glu delivery, however, a strong, statistically significant ABR threshold shift (i.e., hearing loss) was noted at all frequencies, whereas the $\mathrm{H}^{+}$ response remained consistently low. 

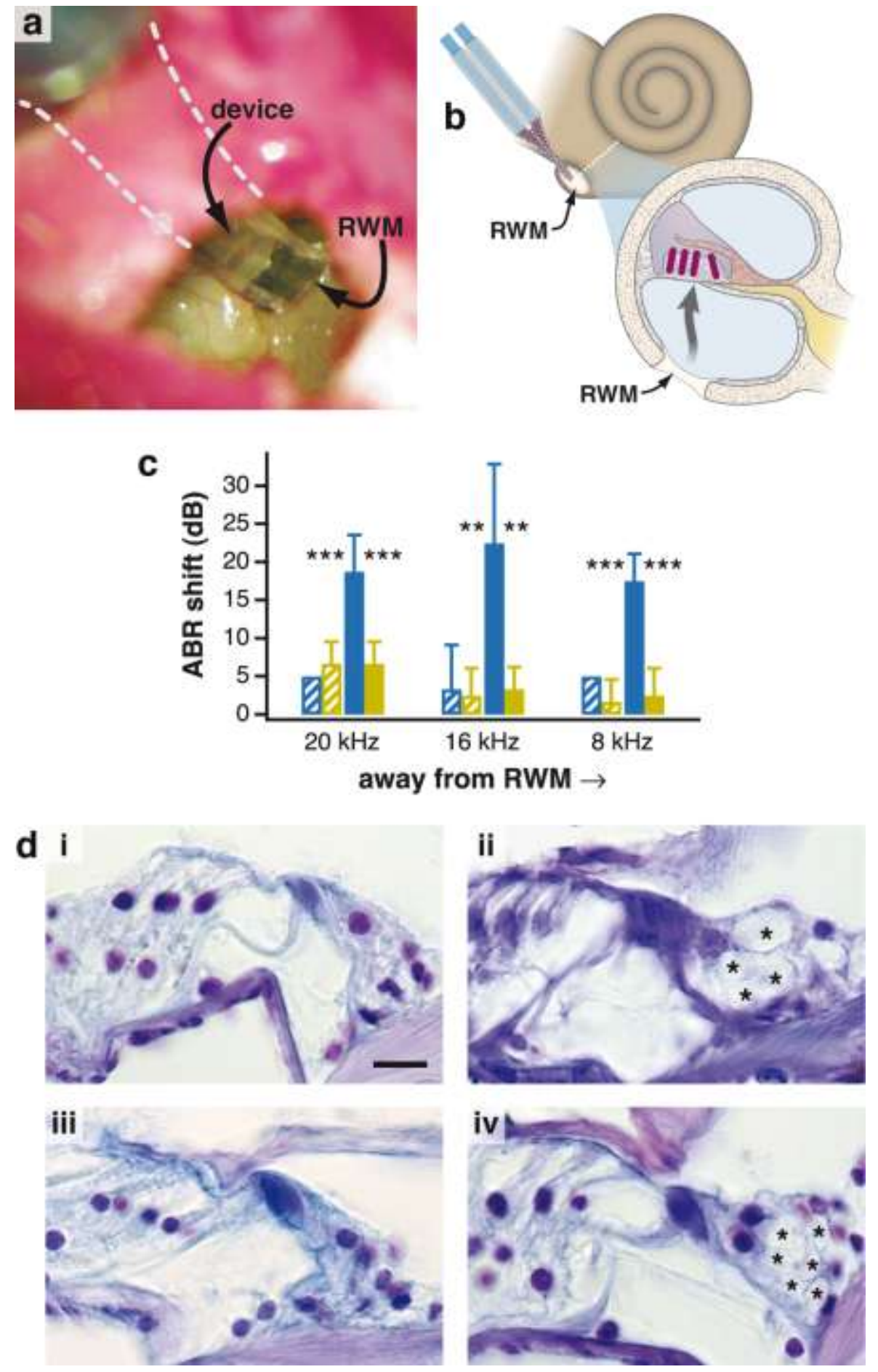

Figure 4 In vivo application of the ion pump. a, Photograph of device mounted on the RWM, with the two ion channels (cf. Figure 1d) visible as dark blue strips on the transparent substrate. The dotted lines indicate the obscured shape of the device leading back to the electrolyte reservoirs, seen to the upper left of the image. b, Illustration of the experimental scheme. The dotted line indicates a slice through the cochlea, expanded below. Here, the gray arrow indicates the diffusion of ions through the RWM, scala tympani, and finally to the hair cells. c, Mean ABR shift (re:pre-treatment thresholds) as a function of recording frequency at $15 \mathrm{~min}$ (dashed bars) and $60 \mathrm{~min}$ (solid bars) into delivery of Glu (blue) and $\mathrm{H}^{+}$(yellow). The frequencies are illustrated in relation to their increasing distance from the RWM. Error bars indicate the standard deviation. e, Histological sections of the cochlea with the inner hair cells on the right and outer hair cells on the left showing (i) effect of $\mathrm{H}^{+}$delivery (as control) and (ii) effect of Glu delivery (excitotoxic-induced damage to the auditory dendrites indicated with asterisks) in the lower basal region (turn 1), and similar (iii) $\mathrm{H}^{+}$and (iv) Glu effect in the upper basal region (turn 2). Scale bar: $20 \mu \mathrm{m}$. 
After the ABR recordings, cochleae were removed and prepared for histological analysis using cresyl violet (Figure 4d). Control experiments revealed that delivered $\mathrm{H}^{+}$had little or no effect on any cell type. Similarly, no morphological changes were observed in the most apical regions of the cochlea after Glu was introduced into the perilymph (Supplementary Figure 1). However, in the lower and upper basal region, a strikingly large number of the dendrites of the inner, but not the outer hair cells, were observed to be swollen. Since these dendrites represent the major connection between the inner hair cells and the underlying auditory nerves, the damaging, excitotoxic effect of Glu explains the corresponding ABR shifts at these frequencies. Collectively, these data indicate that the encapsulated ion pump can be used to establish concentration gradients within the perilymph, providing a means to spatially control excitotoxicity. Furthermore, this demonstrates that delivery of a particular neurotransmitter from the device can be used to selectively target a specific cell type in vivo, as responses are induced solely in the subset of cells expressing the relevant receptor.

Electronic control provides another highly desirable feature: the ability to expose cells to pulsed, rather than continuous delivery. Sustained exposure to neurotransmitters quickly desensitizes the receptors, reflected in, e.g., malfunctioning signalling pathways, or downregulation of receptor expression. Using pulsed delivery, such problems can be circumvented. In the present experiment, $\sim 14 \mathrm{nmol}$ of Glu was delivered during the $60 \mathrm{~min}$ "pulse". The device could accordingly be pulsed more than 100 times without depleting the $\sim 30 \mu$ l Glu solution. Tailoring the pulsing scheme according to the physiological requirements could increase the effective lifetime even further.

Having demonstrated the ability to translate electronic addressing signals, via neurotransmitter signalling, into brainstem responses, this technology establishes a new paradigm in machine-to-brain interfacing. Indeed, the device described herein is the first successful realization of an organic electronic device capable of modulating mammalian sensory function by precise delivery of neurotransmitters. These developments represent a significant 
step forward in biology-technology interfacing, and promise to pioneer further symbiosis of electronics and living systems.

\section{Methods}

Planar ion pump devices were fabricated using standard photolithographic methods as previously reported $^{18}$. Briefly, prefabricated films of PEDOT:PSS on a PET substrate were dry-etched, selectively (by way of photolithographic masking) chemically over-oxidized, and selectively (again by photolithographic masking) coated with the hydrophobic photoresist SU-8. After soaking in deionized water for $>12 \mathrm{~h}$, Glu, Asp, and GABA were transported at various driving voltages between $2 \mathrm{~V}$ and $10 \mathrm{~V}$ for a variety of times at each voltage from $0.1 \mathrm{M}$ source electrolytes. Individual devices were used for each set of parameters. The transport was characterized in terms of the current versus time, as measured via the custom control electronics, and the quantity of the delivered molecules, as determined by quantitative HPLC analysis of the target electrolyte. Device efficiency was thus evaluated as the ratio of electrons passed through the driving circuitry to the number of molecules delivered to the target electrolyte.

Encapsulated ion pump devices were fabricated in a similar fashion as the planar devices. Devices were cut from the substrate using a computer-controlled plotter-table cutter, and sheathed with two pieces of silicone tubing each. The delivery-end of each tube was sealed and much of the length of the delivery "tip" was covered with the ion-impermeable silicone encapsulant Sylgard 186 (Dow Corning).

For in vitro testing, primary murine astrocyte cultures were prepared and maintained as previously described ${ }^{35}$, then loaded with the $\mathrm{Ca}^{2+}$-sensitive dye FURA-2 AM. The encapsulated device was evaluated by delivering Glu and observing the subsequent fluxes in intracellular 
$\left[\mathrm{Ca}^{2+}\right]$ via ratiometric time-lapse fluorescence microscopy. As a control, Glu was also applied manually using a pipette to a final concentration of $1 \mathrm{~m} M$.

For in vivo testing of the encapsulated device, access to the RWM of the anesthetized guinea pig was achieved by a retro-auricle approach. Once the device was placed on the RWM, delivery using either $0.1 M L$-glutamic acid in $10 \mu M \mathrm{HCl}(\mathrm{aq})(\mathrm{pH} \sim 3$ ) or $0.1 M \mathrm{HCl}(\mathrm{aq})(\mathrm{pH} 1)$ as source electrolyte and $0.1 \mathrm{M} \mathrm{NaCl}(\mathrm{aq})$ as cathodic electrolyte was initiated. Control experiments delivering $\mathrm{H}^{+}$utilised $0.1 \mathrm{MHCl}(\mathrm{aq})(\mathrm{pH}$ 1) as source electrolyte. Over a $60 \mathrm{~min}$ timeframe, auditory function was assessed via auditory brainstem response at frequencies of 8 , 16 and $20 \mathrm{kHz}$ at $0,15,30$ and 60 min timepoints. Afterwards, cochleae were collected for histological analysis and stained with cresyl violet.

Full methods are available in Supplementary Methods online.

Acknowledgements We wish to thank Dr. S. Plantman for providing primary astrocyte cultures, Dr. J. Kehr for access to the HPLC equipment, Mrs. A. Viberg for technical assistance, and Dr. D. Nilsson, Dr. P. Kjäll and Prof. T. Nakagawa for valuable discussion. This project has been performed within the Strategic Research Center for Organic Bioelectronics (OBOE, www.oboe.nu) funded by the Swedish Foundation for Strategic Research (SSF). The Organic Electronics Group at Linköping University in Norrköping is a member of the COE@COIN project, also funded by the SSF. B.C. is supported from the Swedish Research Council, Funds of Karolinska Institutet, and Tysta Skolan. The agencies that have supported this study were not involved in the design, interpretation, analysis, or review of the data.

Author contributions D.T.S. was responsible for the design, characterization, and operation of the encapsulated device, and primary preparation of the manuscript. S.K. characterized the planar devices, and K.C.L. performed in vitro cell experiments and assisted with operation of the devices in vivo. R.H. and B.C. performed the surgical procedure, and subsequent audiological and physiological analysis. K.T. manufactured the planar devices, M.G. 
performed HPLC analysis, and both K.T. and E.W.H.J. aided in the design of the encapsulated device. M.B., B.C., and A.R-D. are the senior authors of the paper. Each was responsible for supervision in their respective departments, as well as project planning and preparation of the manuscript.

Additional information Supplementary Information accompanies this paper on www.nature.com/naturematerials. Reprints and permissions information is available online at http://npg.nature.com/reprintsandpermissions. Correspondence should be addressed to A.R-D.

Competing interests statement The authors declare that they have no competing financial interests. 
${ }^{1}$ Wilson, Blake S. et al., Better speech recognition with cochlear implants. Nature $\mathbf{3 5 2}$, 236 (1991).

${ }^{2}$ Kumar, R. et al., Double-blind evaluation of subthalamic nucleus deep brain stimulation in advanced Parkinson's disease. Neurology 51, 850-855 (1998).

${ }^{3}$ Mayberg, H. et al., Deep Brain Stimulation for Treatment-Resistant Depression. Neuron 45, 651-660 (2005).

${ }^{4}$ Hochberg, Leigh R. et al., Neuronal ensemble control of prosthetic devices by a human with tetraplegia. Nature 442, 164-171 (2006).

${ }^{5}$ George, P. et al., Fabrication and biocompatibility of polypyrrole implants suitable for neural prosthetics. Biomaterials 26, 3511-3519 (2005).

${ }^{6}$ Williams, J. C., Holecko, M. M., Massia, S. P., Rousche, P., and Kipke, D. R., Multi-site incorporation of bioactive matrices into MEMS-based neural probes. Journal of Neural Engineering 2, L23-L28 (2005).

${ }^{7}$ Richardsonburns, S. et al., Polymerization of the conducting polymer poly(3,4ethylenedioxythiophene) (PEDOT) around living neural cells. Biomaterials 28, 1539-1552 (2007).

${ }^{8}$ Green, R., Lovell, N., Wallace, G., and Poolewarren, L., Conducting polymers for neural interfaces: Challenges in developing an effective long-term implant. Biomaterials 29, 3393-3399 (2008).

${ }^{9}$ Abhyankar, V., Lokuta, M., Huttenlocher, A., and Beebe, D., Characterization of a membranebased gradient generator for use in cell-signaling studies. Lab on a Chip 6, 389-393 (2006).

${ }^{10}$ Guimard, N., Gomez, N., and Schmidt, C., Conducting polymers in biomedical engineering. Progress in Polymer Science 32, 876-921 (2007). 
${ }^{11}$ Hendricks, J., Chikar, J., Crumling, M., Raphael, Y., and Martin, D., Localized cell and drug delivery for auditory prostheses. Hearing Research 242, 117-131 (2008).

${ }^{12}$ Xu, H., Wang, C., Wang, C., Zoval, J., and Madou, M., Polymer actuator valves toward controlled drug delivery application. Biosensors and Bioelectronics 21, 2094-2099 (2006).

${ }^{13}$ Zinger, Baruch and Miller, L. L., Timed release of chemicals from polypyrrole films. Journal of the American Chemical Society 106, 6861-6863 (1984).

${ }^{14}$ Pernaut, J. and Reynolds, J. R., Use of Conducting Electroactive Polymers for Drug Delivery and Sensing of Bioactive Molecules. A Redox Chemistry Approach. Journal of Physical Chemistry B 104, 11 (2000).

15 Abidian, M. R., Kim, D., and Martin, D. C., Conducting-Polymer Nanotubes for Controlled Drug Release. Advanced Materials 18, 405-409 (2006).

${ }^{16}$ Pei, Q., Pei, Q., Inganaes, O., and Inganaes, O., Electrochemical applications of the bending beam method. 1. Mass transport and volume changes in polypyrrole during redox. Journal of Physical Chemistry 96, 10507-10514 (1992).

17 Wang, X., Shapiro, B., and Smela, E., Visualizing Ion Currents in Conjugated Polymers. Advanced Materials 16, 1605-1609 (2004).

18 Isaksson, J. et al., Electronic control of $\mathrm{Ca}^{2+}$ signalling in neuronal cells using an organic electronic ion pump. Nature Materials 6, 673-679 (2007).

${ }^{19}$ Chiang, C. et al., Electrical Conductivity in Doped Polyacetylene. Physical Review Letters 39, 1098-1101 (1977).

${ }^{20}$ Heywang, G. and Jonas, F., Poly(alkylenedioxythiophene)s - new, very stable conducting polymers. Advanced Materials 4, 116-118 (1992). 
${ }^{21}$ Groenendaal, L., Jonas, F., Freitag, D., Pielartzik, H., and Reynolds, J. R., Poly(3,4ethylenedioxythiophene) and Its Derivatives: Past, Present, and Future. Advanced Materials 12, 481-494 (2000).

${ }^{22}$ Zhu, Z. et al., A simple poly(3,4-ethylene dioxythiophene)/poly(styrene sulfonic acid) transistor for glucose sensing at neutral pH. Chemical Communications, 1556-1557 (2004).

${ }^{23}$ Isaksson, J. et al., Electronically controlled $\mathrm{pH}$ gradients and proton oscillations. Organic Electronics 9, 303-309 (2008).

${ }^{24}$ Riedel, G., Platta, B., and Micheau, J., Glutamate receptor function in learning and memory. Behavioural Brain Research 140, 1-47 (2003).

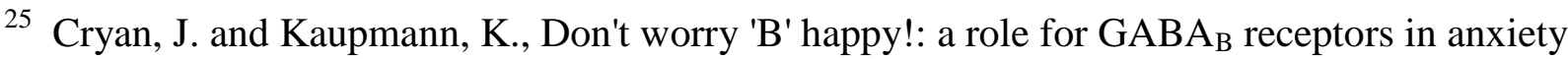
and depression. Trends in Pharmacological Sciences 26, 36-43 (2005).

${ }^{26}$ Meldrum, B. S., Glutamate as a Neurotransmitter in the Brain: Review of Physiology and Pathology 1. Journal of Nutrition 130, 1007S-1015S (2000).

27 Sontheimer, H., Kettenmann, H., Backus, K. H., and Schachner, M., Glutamate opens $\mathrm{Na}^{+} / \mathrm{K}^{+}$ channels in cultured astrocytes. Glia 1, 328-336 (1988).

${ }^{28}$ Usowicz, M. M., Gallo, V., and Cull-Candy, S. G., Multiple conductance channels in type-2 cerebellar astrocytes activated by excitatory amino acids. Nature 339, 380-383 (1989).

${ }^{29}$ Glaum, S. R., Holzwarth, J. A., and Miller, R. J., Glutamate Receptors Activate $\mathrm{Ca}^{2+}$ Mobilization and $\mathrm{Ca}^{2+}$ Influx Into Astrocytes. Proceedings of the National Academy of Sciences 87, 3454-3458 (1990).

30 The Auditory System, in Neuroscience, 4th Edition (ed. Purves, D.) 283-314 (Sinauer, Sunderland, MA, 2004). 
31 Pujol, R., Lenoir, M., Robertson, D., Eybalin, M., and Johnstone, B., Kainic acid selectively alters auditory dendrites connected with cochlear inner hair cells. Hearing Research 18, 145-151 (1985).

32 Duan, M., Agerman, K., Ernfors, P., and Canlon, B., Complementary roles of neurotrophin 3 and a N-methyl-d-aspartate antagonist in the protection of noise and aminoglycoside-induced ototoxicity. Proceedings of the National Academy of Sciences 97, 7597-7602 (2000).

33 Bianchi, L. and Raz, Y., Methods for Providing Therapeutic Agents to Treat Damaged Spiral Ganglion Neurons. Current Drug Targets: CNS \& Neurological Disorders 3, 195-199 (2004).

${ }^{34}$ Ohyama, K., Salt, A., and Thalmann, R., Volume flow rate of perilymph in the guinea-pig cochlea. Hearing Research 35, 119-129 (1988).

35 Dobrenis, K., Microglia in Cell Culture and in Transplantation Therapy for Central Nervous System Disease. Methods 16, 320-344 (1998). 